\title{
Gestational diabetes mellitus and type 2 diabetes: same disease in a different moment of life? Maybe not
}

Lenita Zajdenverg' ', Carlos Antonio Negrato²

${ }^{1}$ Serviço de Nutrologia e Diabetes, Departamento de Clínica Médica, Universidade Federal do Rio de Janeiro (UFRJ), Rio de Janeiro, RJ, Brasil ${ }^{2}$ Associação dos Diabéticos de Bauru, Bauru, SP, Brasil

Correspondence to: Lenita Zajdenverg Serviço de Nutrologia Rua Professor Rodolpho Paulo Rocco, 255 ,

sala 9E14, $9^{\circ}$ andar

Cidade Universitária, Ilha do Fundão 21941-913 - Rio de Janeiro, RJ, Brasil lenitazaj@gmail.com

Received on May/27/2017 Accepted on May/27/2017

DOI: 10.1590/2359-3997000000276
G estational diabetes mellitus (GDM) is a medical condition that has motivated I many debates in the last decades regarding its etiology, pathophysiology, diagnosis, treatment and long-term consequences to the mother and to the fetus.

Women who develop GDM present a metabolic condition similar to that found in type 2 diabetes (T2D) characterized by insulin resistance associated with inadequate insulin secretion (1). Due to similar pathophysiologic mechanisms found between T2D and GDM, there is a great interest in finding markers that will lead to the understanding of a possible common origin to both diseases. Women with GDM also present an inflammatory state that, together with insulin resistance can alter placental gene transcription and many features of fetal programming, that can lead to the development of several metabolic diseases later in life such as glucose intolerance, metabolic syndrome and also a high risk of presenting cardiovascular disease. Women with GDM have sevenfold higher risk of having T2D in the future (2).

Identifying risk markers for the development of GDM or for poor perinatal outcomes will allow the implementation of precocious preventive or therapeutic interventions. Recently, several biomarkers have been evaluated in order to establish this possible relationship such as cord blood adiponectin, C-reactive protein (CRP), advanced glycation end products (AGEs) and a variety of genetic polymorphisms.

Adiponectin exhibits an anti-inflammatory action and may potentially play a protective role in the development of GDM and T2D. Data regarding the relationship between cord blood levels of adiponectin, newborns birth weight and children adiposity are contradictory, with some studies finding a positive correlation (3) and others not showing any correlation (4).

In the present issue of the "Archives of Endocrinology and Metabolism" in a study conducted by Aramesh and cols. in Iran, 52 women with GDM and 52 with normal glucose tolerance (NGT) were evaluated regarding fetal anthropometric parameters, cord blood adiponectin and CRP. It was found that adiponectin levels were higher in the presence of GDM and was also associated with higher birth weight and later gestational ages. The levels of CRP were not different between the two groups (5). This finding contrasts with most studies associating low levels of adiponectin and increased levels of CRP with the risk of progression to T2D (6). Also published in this issue of "Archives of Endocrinology and Metabolism", Lobo Jr. and cols. performed a study with 442 Euro-Brazilian women of which 225 had GDM and 217 presented NGT. Their study had the objective of evaluating the use of serum AGEs as a screening tool for GDM (7). It is well known that AGEs concentrations are associated with several diseases including type 1 and type 2 diabetes mainly in the presence of diabetes-related chronic complications (8). It is supposed that 
high oxidative stress conditions are associated with inflammation and the presence of diabetes. According to the authors, in their study, women with GDM had a good glycemic control which could have influenced the final results. They did not find different AGEs concentrations in GDM, possibly due to the mild severity and short duration of hyperglycemia. It is possible that in this environment they do not generate enough serum AGEs to make it possible discriminate between GDM and NGT groups (7).

The third study published in this issue refers to a cohort of Euro-Brazilian Caucasians formed by 252 patients, 127 with GDM and 125 with NGT. AnghebemOliveira and cols. evaluated the polymorphisms of several genetic variants that are associated with T2D. The authors studied gene polymorphisms T2Drelated such as fat mass and obesity-associated (FTO), leptin receptor (LEPR), peroxisome proliferatoractivated receptor gamma (PPAR $\gamma)$, and transcription factor 7-like 2 (TCF7L2) (9). These polymorphisms are related to food intake, energy balance, appetite regulation, gene expression transcription, glucose and lipids metabolism, inflammation and proliferation of pancreatic beta cells. Some of TCF7L2 polymorphisms have been found to be associated with GDM in other populations (10-13). The authors have found no relation between these polymorphisms with GDM in this Brazilian studied population (9).

The association between previous diagnoses of GDM with high risk of developing T2D is well known (2). Moreover, the screening of diabetes during pregnancy can lead to the discovery of an undiagnosed patient with T2D.

Although the reduction in insulin sensitivity and impaired insulin secretion occur similarly in cases of GDM and T2D, the dysglycemia found in GDM is generally transitory and disappears after delivery. However, the evaluation of non-pregnant women with glucose intolerance that participated in the Diabetes Prevention Program Outcomes Study, followed for ten years, has shown that those that had a history of GDM presented an increased risk of developing T2D when compared to those without previous GDM; this finding was independent of age and BMI. Interestingly, the reduction in the progression to T2D was found only in the group that had had GDM and were treated with metformin (14).

It is also important to note that although hyperglycemia that is first diagnosed during pregnancy is named GDM, it is known that a small percentage of these patients will require further reclassification. The most common types of monogenetic diabetes are frequently diagnosed by the first time during antenatal follow-up (15). Recently, AnghebemOliveira and cols., which did not find the presence of the gen polymorphisms associated with T2D and obesity, has found in this same Brazilian population of pregnant women a higher frequency of carriers of the polimorphism of the $\mathrm{C}$ allele of rs780094 from the glucokinase regulatory protein (GCKR) gen in the group of women with GDM (16). The inclusion, even of a small group of pregnant women probably with monogenetic diabetes, that is not associated with the same pathophysiologic mechanisms of T2D, can interfere with the interpretation of results in studies with a small number of patients.

Dysglycemia found in pregnancy can have different origins and complexities that may not be related to $\mathrm{T} 2 \mathrm{D}$, as proposed by the three manuscripts that were published in this periodic. Further studies are still required to find possible markers for GDM and T2D in order to discover the link that may exist or not between the two conditions.

Disclosure: Lenita Zajdenverg - Advisory board of Novo Nordisk Brazil, Sanofi Brazil and Lilly Brazil. Carlos Antonio Negrato - No disclosures to declare.

\section{REFERENCES}

1. Buchanan TA, Xiang AH. Gestational diabetes mellitus. J Clin Invest. 2005;115(3):485-91.

2. Bellamy L, Casas JP, Hingorani AD, Williams D. Type 2 diabetes mellitus after gestational diabetes: a systematic review and metaanalysis. Lancet. 2009;373(9677):1773-9.

3. Mantzoros CS, Rifas-Shiman SL, Williams CJ, Fargnoli JL, Kelesidis T, Gillman MW. Cord Cord blood leptin and adiponectin as predictors of adiposity in children at 3 years of age: a prospective cohort study. Pediatrics. 2009;123(2):682-9.

4. Mantzoros C, Petridou E, Alexe D, Skalkidou A, Dessypris N, Papathoma $\mathrm{E}$, et al. Serum adiponectin concentrations in relation to maternal and perinatal characteristics in newborns. Eur J Endocrinol. 2004;151(6):741-6.

5. Aramesh MR, Dehdashtian M, Malekian A, ShahAli S, Shojaei K. Relation between fetal anthropometric parameters and cord blood adiponectin and high-sensitivity C-reactive protein in gestational diabetes mellitus. Arch Endocrinol Metab. 2017;61(3):228-32.

6. Chenxiao Liu, Xiu Feng, Qi Li, Ying Wang, Qian Li, Majian Hua. Adiponectin, TNF- $\alpha$ and inflammatory cytokines and risk of type 2 diabetes: A systematic review and meta-analysis. Cytokine. 2016;86:100-9.

7. Lobo Jr JP, Brescansin CP, Santos-Weiss ICR, Welter M, de Souza EM, Rego FGM, et al. Serum Fluorescent Advanced Glycation 
End (F-AGE) products in gestational diabetes patients. Arch Endocrinol Metab. 2017;61(3):233-7.

8. Yamagishi S. Role of advanced glycation end products (AGEs) and receptor for AGEs (RAGE) in vascular damage in diabetes. Exp Gerontol. 2011;46(4):217-24.

9. Anghebem-Oliveira MI, Martins BR, Alberton D, Ramos EAS, Picheth G, Rego FGM. Type 2 diabetes-associated genetic variants of FTO, LEPR, PPARg, and TCF7L2 in gestational diabetes in a Brazilian population. Arch Endocrinol Metab. 2017;61(3):238-48.

10. Pappa KI, Gazouli M, Economou K, Daskalakis G, Anastasiou $\mathrm{E}$, Anagnou NP, et al. Gestational diabetes mellitus shares polymorphisms of genes associated with insulin resistance and type 2 diabetes in the Greek population. Gynecol Endocrinol. 2011;27(4):267-72.

11. Shaat N, Lernmark A, Karlsson E, Ivarsson S, Parikh H, Berntorp $\mathrm{K}$, et al. A variant in the transcription factor 7-like 2 (TCF7L2) gene is associated with an increased risk of gestational diabetes mellitus. Diabetologia. 2007;50(5):972-9.

12. Freathy RM, Hayes MG, Urbanek M, Lowe LP, Lee H, Ackerman $\mathrm{C}$, et al. Hyperglycemia and Adverse Pregnancy Outcome (HAPO) study: common genetic variants in GCK and TCF7L2 are associated with fasting and postchallenge glucose levels in pregnancy and with the new consensus definition of gestational diabetes mellitus from the International Association of Diabetes and Pregnancy Study Groups. Diabetes. 2010;59(10):2682-9.

13. Lauenborg J, Grarup N, Damm P, Borch-Johnsen K, Jorgensen $\mathrm{T}$, Pedersen $\mathrm{O}$, et al. Common type 2 diabetes risk gene variants associate with gestational diabetes. J Clin Endocrinol Metab. 2009;94(1):145-50.

14. Aroda VR, Christophi CA, Edelstein SL, Zhang P, Herman WH, Barrett-Connor E, et al.; Diabetes Prevention Program Research Group. The effect of lifestyle intervention and metformin on preventing or delaying diabetes among women with and without gestational diabetes: the Diabetes Prevention Program outcomes study 10-year follow-up. J Clin Endocrinol Metab. 2015;100(4):1646-53.

15. Chakera AJ, Spyer G, Vincent N, Ellard S, Hattersley AT, Dunne FP. The $0.1 \%$ of the population with glucokinase monogenic diabetes can be recognized by clinical characteristics in pregnancy: the Atlantic Diabetes in Pregnancy cohort. Diabetes Care. 2014;37(5):1230-6.

16. Anghebem-Oliveira MI, Webber S, Alberton D, de Souza EM, Klassen G, Picheth G, et al. The GCKR Gene Polymorphism rs780094 is a Risk Factor for Gestational Diabetes in a Brazilian Population. J Clin Lab Anal. 2017 Mar;31(2). doi: 10.1002/jcla. 22035. 